\title{
Theory of combined AM and high-harmonic FM mode locked laser
}

\author{
ANAND V RAMAMURTHI* and K P J REDDY ${ }^{\dagger}$ \\ "Department of Electrical Communication Engineering, \\ ${ }^{\dagger}$ Department of Aerospace Engineering, \\ Indian Institute of Science, Bangalore 560 012, India \\ Email: "rva@protocol.ece.iisc.ernet.in; ${ }^{\dagger}$ laser@aero.iisc.ernet.in
}

MS received 28 July 1998

\begin{abstract}
Closed form solutions for a simultaneously AM and high-harmonic FM mode locked laser system is presented. Analytical expressions for the pulsewidth and pulsewidth-bandwidth products are derived in terms of the system parameters. The analysis predicts production of $17 \mathrm{ps}$ duration pulses in a Nd:YAG laser mode locked with AM and FM modulators driven at $80 \mathrm{MHz}$ and 1.76 $\mathrm{GHz}$ for $1 \mathrm{~W}$ modulator input power. The predicted values of the pulsewidth-bandwidth product lie between the values corresponding to the pure AM and FM mode locking values.
\end{abstract}

Keywords. Laser; mode-locking; picosecond pulse; pulse compression.

PACS Nos $42.60 ; 42.65 ; 42.55$

\section{Introduction}

The mode locking of lasers for the production of high peak power ultrashort light pulses is the most widely investigated phenomenon in recent times. Demand for the pulses of durations ranging from picosecond to femtoseconds has resulted in the development of innovative techniques which evolved parallel to the development of broadband laser systems [1]. Most commonly used techniques are the active [2], passive [3], synchronously pumped [4], additive pulse [5] and Kerr lens [6] mode-locking.

Stability of the mode-locking process is one of the important features which ensures the repeatability of the characteristics of individual pulses in a train of mode locked pulses. Although active mode locking is not comparable to other techniques in producing short pulses, the study of this technique is still being pursued due to its high repeatability and its suitability for low-power laser systems and gain media. Hence many efforts have been made to enhance pulse compression efficiency of this technique while retaining its high stability [7-9]. Based on earlier proposals [10] recently a new approach to overcome the inherent limitations of the active mode locking has been reported [11]. It is shown that by 
combining the AM mode locking at the cavity fundamental repetition rate with FM mode locking at a high-harmonic, better pulse compression (characterised by high-harmonic mode locking) with higher peak powers (characterised by the mode locking at fundamental repetition rate) can be simultaneously achieved in a laser system.

In this paper we present the theoretical analysis of a laser system mode locked simultaneously by AM modulator at fundamental cavity repetition rate and FM modulator at higher harmonics of the cavity repetition rate. Since in an actively mode locked laser with a single active mode locker the pulsewidth depends weakly on the drive power to the mode locker while its dependence is stronger on the modulation frequency, shorter pulses can be obtained by driving the mode locker at higher frequencies. However, the increase in the modulation frequency leads to the reduction in the pulse peak power as the pulse repetition rate increases. This problem can be eliminated by employing AM mode locking driven at the fundamental cavity repetition frequency and FM mode locking at higher harmonic modulation frequency, where the pulse repetition rate is controlled by the AM mode locking while the FM mode locking controls the pulse compression. Thus the advantages of both types of mode locking can be obtained in this system. The details of the theoretical model are given in $\S 2$ and the results are presented in $\S 3$. Final conclusions are presented in $\S 4$.

\section{Theoretical analysis}

The mode locked laser system considered in the present analysis is shown schematically in figure 1. The laser system consists of a Fabry-Perot cavity containing a laser medium, an electro-optic FM modulator and an acousto-optic AM modulator. We extend the selfconsistent model, proposed earlier [10] for the actively mode locked laser system, to analyse the pulse compression process in the simultaneously AM-FM mode locked laser. In this model we assume that a laser pulse of Gaussian shape is circulating inside the laser cavity with an electric field in the form $E(t)=\left(E_{0} / 2\right) \exp \left(-\gamma t^{2}\right) \exp \left(j \omega_{p} t\right)$, where $\omega_{p}$ is the center frequency of the pulse spectrum in radians, $E_{0}$ is the pulse amplitude and $\gamma=\alpha-j \beta$ is the shape factor in which $\alpha$ and $\beta$ are related to the pulsewidth and pulsewidth-bandwidth products as $\tau_{p}=\sqrt{(2 \ln 2) / \alpha}$ and $\tau_{p} \Delta f_{p}=(2 \ln 2 / \pi) \sqrt{1+(\beta / \alpha)^{2}}$, respectively. It is assumed that the passage of the laser pulse through the cavity elements will alter only the amplitude while preserving the shape. This is achieved by assuming the laser medium to be homogeneously broadened and the shape of the gain curve in the form

$$
g(\omega)=G \exp \left[-\left(\frac{2 j g}{\Delta \omega}\right)\left(\omega-\omega_{a}\right)-\left(\frac{4 g}{\Delta \omega^{2}}\right)\left(\omega-\omega_{a}\right)^{2}\right]
$$

where, $G=e^{g}, g$ is the saturated round-trip amplitude gain, $\omega_{a}$ is frequency in radians corresponding to the atomic line center, and $\Delta \omega$ is the atomic line width. Here we have assumed that there is no detuning in the system. The transmission functions for the amplitude modulator and the frequency modulator are assumed in the following approximate form to preserve the Gaussian form of the pulse after every passage [10],

$$
a_{1}(t) \cong\left[-2 \delta_{1}\left(\omega_{\mathrm{am}} t\right)^{2}\right] .
$$

for the AM modulator with a modulation index $\delta_{l}$ and

$$
f_{1}(t) \cong \exp \left(\mp j 2 \delta_{c} \pm j \delta_{c}\left(\omega_{\mathrm{fm}} t\right)^{2}\right)
$$




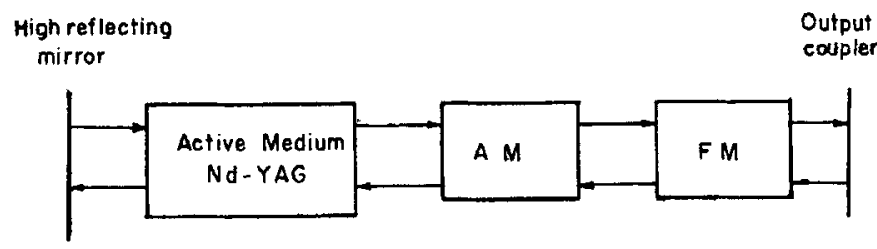

Figure 1. Schematic diagram of simultaneous $A M$ and high-harmonic FM mode locked laser.

for the FM modulator with the modulation index $\delta_{c}$, where $\omega_{\mathrm{am}}$ and $\omega_{\mathrm{fm}}$ are the modulation frequencies in radians of the AM and FM modulators, respectively. Here it is assumed that the pulsewidth is shorter than the modulation period of the FM modulator. The two possible solutions to the FM transmission function correspond to the two extremes of the phase variation.

At steady state the mode locked pulse shape reproduces itself after every round trip through the cavity with a slight phase delay. Thus the net change undergone by the pulse in one round trip at steady state can be equated to zero. Following the pulse through the elements inside the laser cavity and applying self-consistency condition we get the following equations at steady state

$$
\begin{aligned}
& T_{m}=\frac{2 L_{0}}{c}+B, \\
& \gamma=\frac{1}{4 A}+\left(2 \delta_{l} \omega_{\mathrm{am}}^{2}-j \delta_{c} \omega_{\mathrm{fm}}^{2}\right), \\
& \exp (-j \phi)=\frac{r G}{2 \sqrt{r A}} \exp \left(j \omega_{a} B+2 \delta_{c}\right),
\end{aligned}
$$

where $T_{m}$ is the total round trip time for the pulse inside the laser cavity, $L_{0}$ is the length of the laser cavity, $A=\left(1 / 4 \gamma+4 g / \Delta \omega^{2}\right), B=2 g / \Delta \omega, c$ is the velocity of light and is the effective reflectivity of the mirror, which includes all the losses in the cavity. The shape factor $\gamma$ in eq. (1) can be written as $\gamma=1 / 4 A+\delta_{g} \omega_{\text {am }}^{2}$ where, we have substituted $\omega_{\mathrm{fm}}=n \omega_{\mathrm{am}}, \delta_{l}^{\prime} \equiv \delta_{l} / n^{2}, \delta_{g} \equiv n^{2}\left(2 \delta_{l}^{\prime}-j \delta_{c}\right)$ and $n$ is an integer. By including the host medium dispersion term in the above equation we obtain an analytical solution for $\gamma$ in the form

$$
\gamma=\frac{\omega_{\mathrm{am}} \Delta \omega}{4} \sqrt{\frac{\delta_{g}}{g_{0}}}\left(1-j \frac{L_{0}^{\prime \prime} \omega_{a} \Delta \omega^{2}}{4 g_{0} c}\right)
$$

where, $L_{0}^{\prime \prime}=\left(n_{1}^{\prime \prime} / 2\right) l_{c 1}+\left(n_{2}^{\prime \prime} / 2\right) l_{c 2}+\ldots$ is the second order change in the optical path length due to the passage through the crystals of length $l_{c 1}$ and $l_{c 2}$ and $g_{0}$ is the saturated amplitude gain of the host medium at the line center. Substituting the value of $\delta_{g}$ we can rewrite the above equation as

$$
\gamma \cong \frac{n \omega_{\mathrm{am}} \Delta \omega}{4} \sqrt{\frac{1}{g_{0}}}\left[\left(\left(2 \delta_{l}\right)^{2}+\left(\delta_{c}\right)^{2}\right)^{1 / 2}\left(1+\left(\frac{L_{0}^{\prime \prime} \omega_{a} \Delta \omega^{2}}{4 g_{0} c}\right)^{2}\right)\right]^{1 / 2}
$$




$$
\exp \left(j\left(\frac{\theta}{2}-\phi\right)\right)
$$

where, $\theta \equiv \tan ^{-1}\left(\delta_{c} / 2 \delta_{l}\right)$ and $\phi \equiv \tan ^{-1}\left(L_{0}^{\prime \prime} \omega_{a} \Delta \omega^{2} / 4 g_{0} c\right)$. Separating the real and imaginary parts of the shape factor we get the following analytical expressions for the pulsewidth and pulsewidth-bandwidth products of the mode locked pulse

$$
\begin{gathered}
\tau_{p}=\sqrt{2 \ln 2}\left[\left(\left(2 \delta_{l}\right)^{2}+\left(\delta_{c}\right)^{2}\right)^{1 / 2}\left(1+\left(\frac{L_{0}^{\prime \prime} \omega_{a} \Delta \omega^{2}}{4 g_{0} c}\right)^{2}\right)\right]^{-1 / 2} \\
{\left[\frac{4 \sqrt{g_{0}}}{n \omega_{\mathrm{am}} \Delta \omega \cos \left(\frac{\theta}{2}-\phi\right)}\right]^{1 / 2}}
\end{gathered}
$$

and

$$
\tau_{p} \Delta f_{p}=(2 \ln 2 / \pi)\left[1+\left(\frac{\sin \left(\frac{\theta}{2}-\phi\right)}{\cos \left(\frac{\theta}{2}-\phi\right)}\right)^{2}\right]^{1 / 2}
$$

These simple equations can be used to study the parametric dependence of the characteristics of the dual mode locked pulse on the system parameters. Application of the analysis for a typical practical system is presented here.

\section{Results and discussion}

Since it is difficult to modulate the acousto-optic crystal used for AM mode locking beyond $1 \mathrm{GHz}$, we assume that the AM mode locking is achieved at a frequency corresponding to the cavity repetition frequency. Thus the AM mode locking limits the pulse repetition rate by providing a periodic gain window at the fundamental cavity repetition rate. On the other hand since the electro-optic crystal used for FM mode locking can be modulated at much higher frequencies we assume that FM mode locking is achieved at higher harmonic of the cavity repetition rate. The frequency of modulation $f_{\mathrm{fm}}$ for the FM modulator is chosen such that it is less than $\left(\omega_{\mathrm{am}} / 2 \Delta \theta\right)$ where $\Delta \theta$ is the phase angle satisfying the size of the window in radians during which the net gain exceeds the loss inside the laser cavity. This ensures that only one mode locked pulse exists per round trip inside the laser cavity and for the modulation frequencies above this value multiple pulsing occur leading to the unstable operation of the laser [11].

In the present analysis we assume a Nd:YAG laser system with a cavity length of $1.875 \mathrm{~m}$, so that the frequency of modulation of the AM modulator is set at $80 \mathrm{MHz}$ and that of the FM modulator is $1.76 \mathrm{GHz}$, corresponding to the $22^{\text {nd }}$ harmonic of the AM modulation frequency. Both the modulation frequencies are assumed to be generated from a common crystal RF oscillator to ensure the phase locking between the AM and FM mode lockers. The efficiency of the FM modulator is taken to be $\eta=0.59 \mathrm{rad} /(W)^{1 / 2}$. Other system parameters considered are the saturated gain $g=0.1$ and the amplifier linewidth $\Delta \omega=120 \mathrm{GHz}$. The input power to the AM mode locker is adjusted such that it generates mode locked pulses of 75 ps duration at $80 \mathrm{MHz}$ repetition rate in absence of the FM 


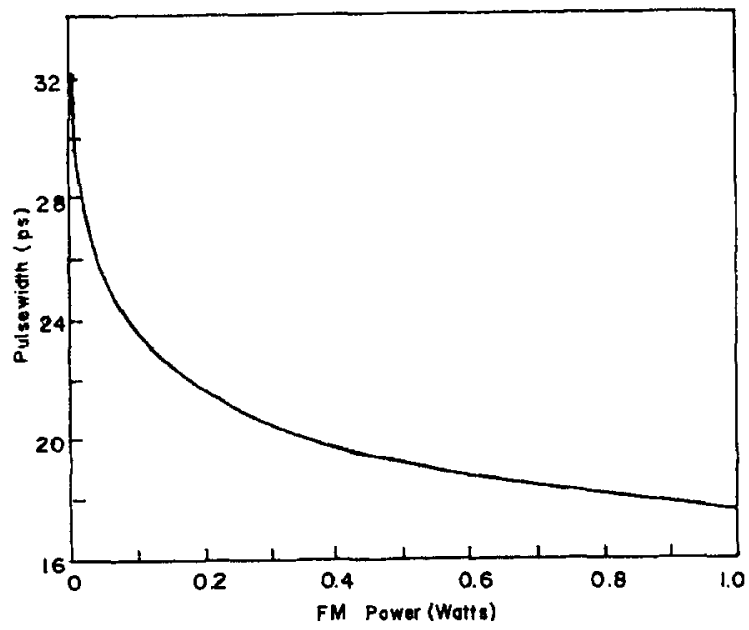

Figure 2. Variation of pulsewidth as a function of the FM mode locker power.

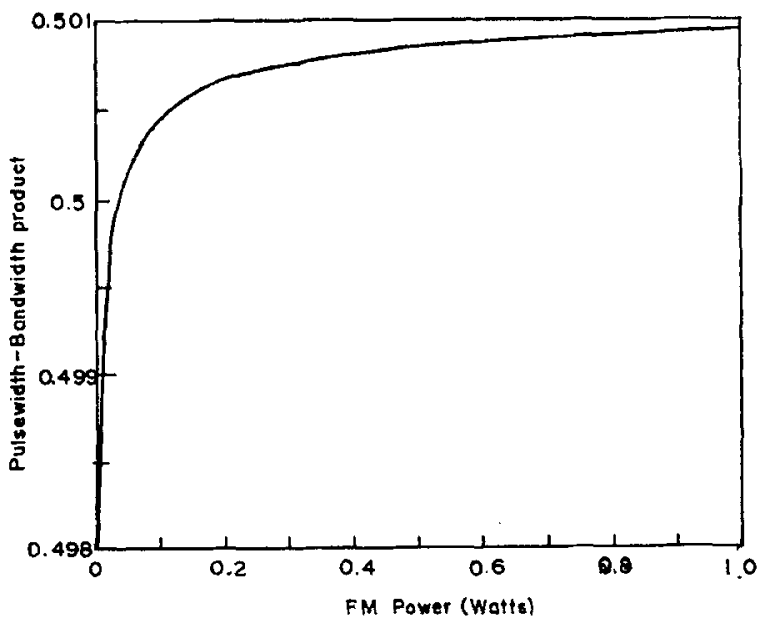

Figure 3. Variation of pulsewidth-bandwidth product as a function of the FM mode locker power.

mode locking. The variation of the mode locked pulsewidth as a function of the input power to the FM mode locker is presented in figure 2. The results presented here show that the decrease in the pulsewidth with the increase of the input power to the FM mode locker is very fast for the power levels below $30 \mathrm{~mW}$. At very low powers the mode locking process is dominated by the AM mode locking and hence the pulsewidth is closer to the pure AM case. As the power of the FM modulator becomes appreciable the pulses get compressed rapidly due to the combined action of the increase in the modulation depth and increase in the effective modulation frequency. However, at power levels above $30 \mathrm{~mW}$ the pulse compression is completely taken over by the FM modulator and the modulation frequency changes from $80 \mathrm{MHz}$ to $1.76 \mathrm{GHz}$. In this region the pulse compression is only due to the 
increase of modulation depth with the increase of the modulator pump power and hence the reduction of the pulsewidth is slow. The results presented here show that pulses of $17 \mathrm{ps}$ duration could be obtained for $1 \mathrm{~W}$ FM modulator power in the present case. The variation of the pulsewidth-bandwidth product with the FM modulator power is shown in figure 3 . The product varies between 0.44 and 0.626 which correspond to the pure AM and FM cases, respectively. The predicted values of the pulsewidth and pulsewidth-bandwidth products match very well with the recently reported experimental values for the Nd:YAG laser system [11].

\section{Conclusion}

The analysis of simultaneous AM and high-harmonic FM mode locked laser is presented. Analytical expressions are obtained for the pulsewidth and pulsewidth-bandwidth products based on the self-consistency theory at steady state. The theory predicts the production of pulses of 17 ps duration in a Nd:YAG laser simultaneously AM and FM mode locked with modulation frequencies of $80 \mathrm{MHz}$ and $1.76 \mathrm{GHz}$, respectively. The predicted values of the pulsewidth-bandwidth product lie between the values for the pure AM and FM mode locking. Although the analysis presented here is for zero detuning, it can be easily extended to include the detuning effects.

\section{References}

[1] J D Simon, Rev. Sci. Instrum. 60, 3597 (1989)

[2] B C Johnson, P F Moulton and A Mooradian, Opt. Lett. 10, 116 (1984)

[3] N Sakura, Y Ishida, H Nakano and Y Yamamoto, Appl. Phys. Lett. 56, 814 (1990)

[4] L F Mollenauer, N D Viera and L Szeto, Opt. Lett. 7, 414 (1982)

[5] J Mark, L Y Liu, K L Hall, H A Haus and E P Ippen, Opt. Lett. 14, 48 (1989)

[6] D E Spence, P N Kean and W Sibbett, Opt. Lett. 16, 42 (1991)

[7] H A Haus and H L Dyckman, Int. J. Electron., 44, 225 (1978)

[8] K P J Reddy, Opt. Commun., 37, 77 (1981)

[9] T S Kinsel, IEEE J. Quantum Electron. 9, 3 (1973)

[10] D J Kuizenga and A E Siegman, IEEE J. Quantum Electron. 6, 694 (1970)

[11] R P Scott, C V Bennett and B H Kolner, Appl. Opt. 36, 5908 (1997) 